\title{
Tumour banks and oncological research
}

In science, every advance is based on technical development and, at least partially limited by new subsidiary technological requirements. Nowadays we are being witnesses of the deepest transformation in Medicine since the beginnings of the scientific mind in the Renaissance era. Current molecular biotechnology is allowing a knowledge about cancer phenomena as never before, and more importantly, all these new data obtained by the basic research are passing to the clinical level through translational research. A new concept of Clinical Oncological Pathology is growing progressively, based on the application of molecular technologies to new and classic clinical questions and on the molecular view of cancer. So, we are moving from a phenomenological study of cancer, mainly based on symptoms and histopathological aspects, to analyse the plethora of molecular changes that define cancer as a biological entity and define each patient individually.

This new Clinical Pathology does not avoid current methods and objectives, which have been the base of a great part of the medical advance occurring in the XXth century, but includes new possibilities and goals for molecular diagnosis of tumours, therapeutic susceptibility, molecular prognosis markers, identification of genes involved in oncogenic susceptibility and identification of relevant genes as possible therapeutic targets. At the same time, translational research requires active communication between basic and clinical researchers. Pathologists can and must play this role, as it already occurred last century.

As it was mentioned previously, technological development is usually limited by other aspects. Progress always creates new needs, and the main new requirement in this new time for the oncological research and care is simply tissue, human tissue suitably processed to allow molecular studies. Thanks to current biotechnology, in a few years and at present, a more accurate nosological and prognostic diagnosis will be possible, with personalised therapy approaches. But it will only be possible if we have high quality frozen samples, i.e.: careful selection, handling, freezing, storage and shipping, mainly when advances in Molecular Pathology have a progressive clinical value.
Current oncological research has three main characteristics: The capacity for large-scale studies in genomics and proteomics mainly by using of techniques such as cDNA arrays, the high sensitivity of the current tools in genomics and proteomics, and the transfer from basic to clinical research. And all these three characteristics are dependent of especially procured tissues: Large-scale molecular studies need large numbers of cases to identify new parameters of clinical value, highly sensitive techniques require samples that have been handled with appropriate procedures, and the transfer from basic to clinical research needs homogeneous tissue-sampling protocols for multi-centre studies. Treatment response prediction must be based on clinical trials. However, these trials must include the acquisition of clinical samples for molecular studies.

But tissue procurement has yet another limitation: A sample is only as good as its initial collection process, and the quality of a tissue sample depends on the handling in its early 15 - 30 minutes. This is the responsibility of Tumour Banks (TB).

Currently TB is defined as an activity involving the entire Hospital, although with the special involvement of the Pathology Department. A Tumour Bank does not only entail tissue storage, but also a series of hospital protocols that allow molecular studies of tumour samples. A Tumour Bank requires collection, freezing and storage of neoplastic and normal tissues and these activities must be considered a routine in the Departments of Pathology in such a way that a TB should be a centrepiece in a Hospital and a Pathology Department's bid for accreditation.

As previously mentioned, current oncological research needs of a large number of cases homogeneously treated, followed up and with tissue samples. There is no Hospital in the world large enough to collect all the necessary casuistic by itself. The same can be said about regions and countries when we refer to polymorphisms and/or rare types of neoplasms. For this reason, networking appears the best environment where TB must grow.

Tumour banks and tumour bank networks are not an aim in themselves... they are only tools, and the value 
of a tool depends on the goals it has been made for, and its adaptation to these goals. For this reason talking about a TB Network is to talk about its goals and limitations. Very frequently TB Networks are planned from a centralised point of view, by the organisation who promotes the network. However, a TB is mainly a hospital activity and must be observed from the hospital point of view.

Another source for difficulties arises when TB is considered only a part of the pathologists' activities. On the contrary, it is a hospital activity affecting not only pathologists but also surgeons, clinicians, hospital managers, etc. Finally, it is evident that TB are powerful tools for research and teaching, but it is critical not to forget that they have very transcendent implications in cancer patients' care.

The CNIO (Centro Nacional de Investigaciones Oncológicas, Spanish National Cancer Centre) is the promoter of the Spanish National Tumour Bank Network (www.cnio.es), a unique design built to instigate high quality TB in Spanish Hospitals and to connect their databases and activities in a co-operative and decentralised network, co-ordinated by the CNIO.

Hospitals associated to the Spanish National Tumour Bank Network (SNTBN), keep their own samples, being their only owner. On the other hand, they are all compromised to use the same homogeneous technical and ethical procedures to assume a unified quality control programme and to accept the centralised co-ordination of the CNIO.
In this way, each Centre's tissue remains in the Hospital itself, thereby playing a key role in the development of the welfare, teaching and research activities within the Hospital, and at the same time, it represents a tool for the encouragement of multi-hospital cancer research. Therefore, the current design is not of a Central Tumour Bank, but rather of a co-operative and co-ordinated Network of Hospital Banks, based on simple, homogeneous and optimal tissue management protocols.

The associated centres have the highest priority to use of the services of the network, as well as to access the technology available in the CNIO and its scientific activities and training of researchers, specialists and technicians. Furthermore, SNTBN activity is not only restricted to associated centres. All the Spanish research groups can be users of the network, independently from being a part of an associated hospital or not, and independently from being basic or applied researches.

This design is guaranteed by the realisation of over than 60 scientific projects in the first two years of activity of the network, and is serving as model for the creation of other national nets in Europe and elsewhere.

M.M. Morente
Spanish National Tumour Bank Network.
Molecular Pathology Programme.
Centro Nacional de Investigaciones Oncológicas -CNIO-.
Madrid.

